\title{
A Values-Tailored Web-Based Intervention for New Mothers to Increase Infant Vaccine Uptake: Development and Qualitative Study
}

Amanda Dempsey ${ }^{1 *}, \mathrm{MD}, \mathrm{MPH}, \mathrm{PhD}$; Bethany M Kwan ${ }^{1 *}, \mathrm{PhD}$; Nicole M Wagner ${ }^{2}, \mathrm{PhD}$; Jennifer Pyrzanowski ${ }^{1}$, MSPH; Sarah E Brewer ${ }^{1}$, PhD, MPA; Carter Sevick ${ }^{1}$, MS; Komal Narwaney ${ }^{2}$, PhD; Kenneth Resnicow ${ }^{3}$, PhD; Jason $\mathrm{Glanz}^{2}, \mathrm{PhD}$

\footnotetext{
${ }^{1}$ University of Colorado, Denver, Aurora, CO, United States

${ }^{2}$ Kaiser Permanente Colorado Institute for Health Research, Denver, CO, United States

${ }^{3}$ University of Michigan, Department of Health Behavior \& Health Education, School of Public Health, Ann Arbor, MI, United States

*these authors contributed equally
}

\section{Corresponding Author:}

Amanda Dempsey, MD, MPH, PhD

University of Colorado, Denver

13199 East Montview Blvd, Suite 300

Aurora, CO, 80045

United States

Phone: 13037246679

Email: amanda.dempsey@ucdenver.edu

\section{Abstract}

Background: Vaccine hesitancy among parents leads to childhood undervaccination and outbreaks of vaccine-preventable disease. As the reasons for vaccine hesitancy are diverse, there is often not enough time during regular clinical visits for medical providers to adequately address all the concerns that parents have. Providing individually tailored vaccine information via the internet before a clinical visit may be a good mechanism for effectively allaying parents' vaccination concerns while also being time efficient. Including tailoring based on values is a promising, but untested, approach to message creation.

Objective: This study aimed to describe the process by which we developed a Web-based intervention that is being used in an ongoing randomized controlled trial aimed at improving the timeliness of infant vaccination by reducing parental vaccine hesitancy.

Methods: Development of the intervention incorporated evidence-based health behavior theories. A series of interviews, surveys, and feedback sessions were used to iteratively develop the intervention in collaboration with vaccination experts and potential end users.

Results: In all, 41 specific content areas were identified to be included in the intervention. User feedback elucidated preferences for specific design elements to be incorporated throughout the website. The tile-based architecture chosen for the website was perceived as easy to use. Creating messages that were two-sided was generally preferred over other message formats. Quantitative surveys identified associations between specific vaccine values and vaccination beliefs, suggesting that values tailoring should vary, depending on the specific belief being endorsed.

Conclusions: Using health behavior theories, qualitative and quantitative data, and significant expert and end user input, we created a novel, Web-based intervention to improve infant vaccination timeliness. The intervention is based on tailoring messages according to each individual's values and beliefs. This intervention is currently being tested in a controlled randomized clinical trial.

(J Med Internet Res 2020;22(3):e15800) doi: 10.2196/15800

\section{KEYWORDS}

immunization; parents 


\section{Introduction}

\section{Vaccine Hesitancy}

Vaccination is widely recognized as one of the most effective public health interventions ever [1]. However, despite the well-established safety and effectiveness of vaccines, a growing number of parents are choosing to delay or forgo them for their children because of questions about vaccines' necessity and safety (also sometimes because of firmly held religious or political beliefs). This phenomenon is referred to as vaccine hesitancy [2,3]. Vaccine hesitancy often results in undervaccination among children and has led to increasing numbers of vaccine-preventable disease outbreaks in the United States over the last two decades [4,5]. For example, measles, which was considered eradicated from the United States in 2000, caused infections among more than 2000 people between 2014 and 2018 alone, with the majority of cases occurring in individuals under- or unvaccinated against the virus [6]. Vaccine-preventable disease outbreaks are associated with significant cost and morbidity, and in some cases, even death [7]. Therefore, it is a public health priority to find interventions to mitigate this trend of parental vaccine hesitancy and childhood undervaccination [8]. This paper reports on the development of a novel, tailored, Web-based intervention to promote timely vaccination by addressing these issues. A more detailed description of the study design and protocol is available elsewhere [9].

\section{Study Rationale}

Most interventions to increase vaccination developed thus far have focused primarily on correcting knowledge deficits, with the hypothesis that correcting these deficits will lead to improved attitudes and behaviors (ie, parents become less vaccine hesitant and thus are more likely to get their children vaccinated). Unfortunately, the majority of interventions based on this concept have not been effective at increasing vaccination rates $[10,11]$. This is because, as research has elucidated, parents' vaccination decisions are multiply determined—based not just on their knowledge about the risk and severity of infectious diseases and the benefits and risks of vaccines but also on trust, emotion, values, past experience, access to health care, and peer influences $[12,13]$.

Given the multiple determinants of vaccination decisions, novel intervention strategies that account for factors beyond knowledge deficits-such as personal values and emotions associated with parents' individual concerns and barriers to vaccination-are needed [14]. One such approach to address these multiple issues is message tailoring. Message tailoring involves providing customized vaccine-promotion messages based on an individual's unique beliefs, experiences, knowledge, and barriers to action [15]. Research on tailored messaging in multiple domains shows that by increasing the personal relevance of the information, people are more receptive to new information that may challenge their beliefs. Regardless of whether their knowledge of a given situation is altered by message tailoring, the technique is thought to work by lowering psychological resistance to information or suggested action that may counter what an individual initially thinks or believes [15].
Tailored messaging interventions have been shown to be effective for a number of health behaviors but have not been extensively tested for vaccine promotion $[16,17]$.

In this paper, we describe the development of a Web-based, tailored messaging intervention used in a randomized controlled trial that is ongoing (ClinicalTrials.gov protocol number NCT02665013). This intervention, targeted to new and expectant mothers, was designed to promote vaccination by either reinforcing provaccination decisions among parents who are not hesitant to vaccinate or by decreasing vaccine hesitancy and thus increasing vaccination intention among parents who are vaccine hesitant. To do this, we developed our intervention based on evidence-based health behavior theories and included tailoring in both standard (ie, gender, primary vaccination beliefs, and concerns) and novel (personal values) domains. Although many additional factors could be novel targets for tailoring (trust, emotion, access to health care, etc), values were chosen as the potential new tailoring target for this intervention because, unlike beliefs, experiences, and barriers, which frequently change over time or by situation, values are believed to remain stable over the life course and across contexts [18-21]. Moreover, the behavior of vaccine hesitancy appears rooted in values as well as knowledge, skills, and self-efficacy [13,22-24]. Although there has been minimal research on how to use values to effectively promote vaccination, many researchers in the field recognize its potential importance [23,25-28]. Thus, a secondary goal in developing this values-tailored intervention was to begin to address the knowledge gap regarding the role values may play in creating messages effective for increasing maternal acceptance of vaccines. In addition, although fathers can be an important influence in childhood vaccination decisions, they were not included as the target for this intervention based on our previous research suggesting that mothers were the main vaccine decision makers in the study population of interest $[29,30]$. In this paper, we describe the overall intervention development process, with particular attention to how the novel values tailoring was developed.

\section{Methods}

\section{Design Overview}

The Web-based, tailored vaccine promotion messaging intervention was developed using a multiphase, iterative, user-centered design process. It was informed by behavioral theory, empirical data from maternal surveys and interviews, and expert and end-user input. When possible, end users' opinions about intervention design were prioritized over those of the research team. For the intervention trial, we planned that mothers would be recruited to the website between the last trimester of their pregnancy and when their child was less than or equal to 2 months of age (the primary time for vaccination decisions to be solidified [30]). Mothers would then receive additional exposures to the website (with retailored information based on updated beliefs) at three additional time points between enrollment and infant age of 15 months. Vaccination timeliness would be assessed at age 200 days (ie, approximately 6 months) and 489 days (ie, approximately 15 months). Two versions of the website were developed for the randomized trial: a version 
with messages tailored to the individual participant based on personal characteristics (age, baby's gender, and pregnancy status) and mothers' vaccination beliefs and concerns, vaccination values, and intention to vaccinate (described in this paper), and an untailored version that was identical in appearance and content to the tailored version except for the tailored components (described elsewhere) [31].

Methods used to establish the tailored website's architecture, content, and tailoring included the following:

1. A conceptual model reflecting empirically supported theories and intervention strategies for attitude and behavior change (Figure 1).
2. Developing informational content for the intervention using data from a previous intervention [32], the published literature, and end-user input.

3. Assessing the architecture of the intervention by evaluating an untailored prototype with usability testing and one-on-one interviews with potential end users of the website.

4. Iteratively developing and testing different message tailoring approaches using (a) survey data that assessed the relationships between maternal values and vaccination beliefs, (b) structured interviews with potential end users of the intervention on different types of message framing approaches in combination with tailored information, and (c) health communication expert and research team consensus.

Figure 1. Conceptual model for a tailored messaging intervention to promote childhood vaccination.

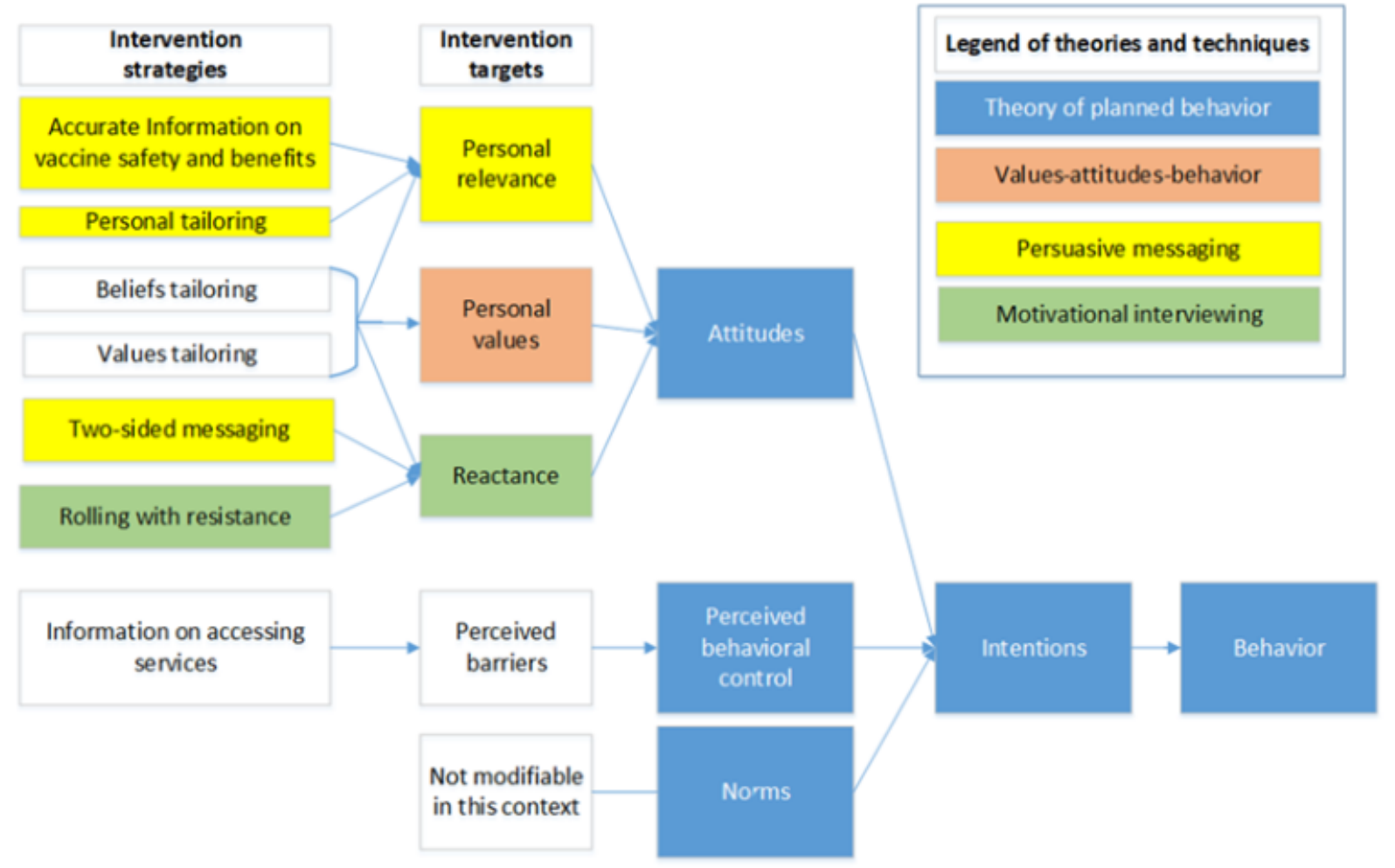

\section{Conceptual Model}

The theoretical framework for this intervention (Figure 1) was a hybrid of the Theory of Planned Behavior (TPB) [33] and the Values-Attitudes-Behavior (VAB) model [34]. According to the TPB, behavior (in this case, childhood vaccination) is directly influenced by intentions (intention to vaccinate), which are based on one's attitudes, perceived norms, and perceived behavioral control. In the TPB, perceived behavioral control is a function of control beliefs (eg, beliefs that the decision to vaccinate is in the parents' power to control) and attitudes are a function of behavioral beliefs (eg, beliefs that vaccinating one's child will prevent infectious disease and will not harm the child). The VAB theory goes beyond the TPB to posit that personal values are factors influencing attitudes-this is the basis for our hypothesis that values-tailored messaging would be an effective behavior change target in the intervention. We also used select principles of motivational interviewing (MI) [35] and persuasive messaging [36,37] to inform intervention design, aiming to increase the personal relevance of the materials while minimizing reactance. Specifically for MI, the idea of rolling with resistance (not directly counteracting a person's antivaccination attitudes for example) is believed to decrease a person's reactance to receiving information that may be counter to their current beliefs [35,38]. Persuasive messaging in this context refers to making the information more personally relevant by tailoring the information to the person's needs and more trustworthy by providing information that is perceived as accurate and balanced (two-sided messaging) with regard to potential risks and harms related to vaccination. 


\section{Setting and Participants}

The setting for this project was Kaiser Permanente Colorado (KPCO). KPCO is a managed care organization in the Denver metropolitan area that maintains an electronic health record with demographic, medical encounter, and vaccination data on all members. Between January 2015 and October 2015, various convenience samples (described below and in Figure 2) of mothers of young children currently enrolled at KPCO were recruited for the design and tailored message testing interviews, usability testing, and surveys.

Figure 2. Study participant flow and activities.

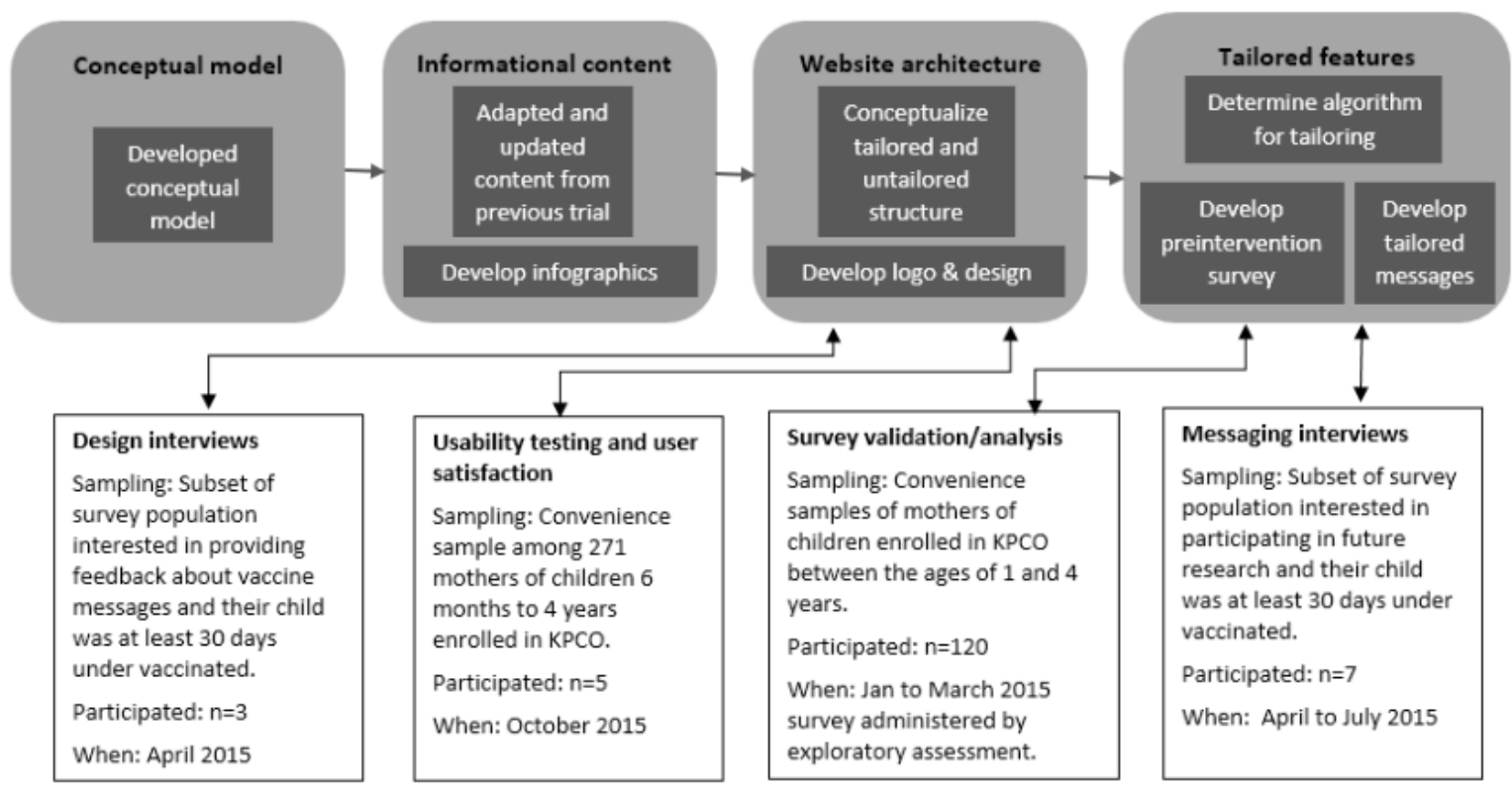

\section{Developing Informational Content}

Informational content for the website was developed by editing content adapted from an intervention used in a previous trial to reflect the most current vaccine information and recommendations [32], and developing new content based on emerging vaccination issues identified by vaccine experts and from vaccine questions received from parents in a previous trial [39]. When possible, content was contextualized to the local Colorado environment and health system (ie, reflected vaccines in the KPCO vaccine formulary) and was written at an eighth-grade reading level. All information presented was evidence based and included updated references from peer-reviewed journal articles and materials from the Centers for Disease Control and Prevention.

\section{Assessing and Finalizing the Architecture of the Intervention}

\section{Design Interviews, Usability Testing, and User Satisfaction}

We solicited feedback from parents of children aged 1 to 2 years on preliminary color schemes, logos, and general architecture of the intervention using printed prototypes. These parents were recruited based on a prior indication of interest in participating in the intervention's development when participating in the values survey described in the following sections.

On the basis of their feedback, we created an interactive, untailored, Web-based prototype to assess usability and user

satisfaction. Using the electronic health record, we identified a random sample of 271 English-speaking mothers of children aged 6 months to 4 years currently enrolled in KPCO and not part of the previous study [32]. These individuals were recruited via email. Usability was assessed using the think aloud methodology, in which users provided verbal feedback as they completed specific tasks, such as logging on to the website and identifying specific types of information. User satisfaction was then assessed using the System Usability Scale (SUS) that includes 10 questions measured on a 5-point Likert scale [40]. SUS scores above 70 (total possible range $0-100$ ) are considered passable. This feedback was incorporated to create the final version of the tailored (and untailored) websites.

\section{Iterative Development of Tailored Messages Incorporating Values and Framing}

To create a personalized Web-based experience for each participant, the intervention was designed such that a preintervention survey would assess individuals' values, beliefs, and vaccine hesitancy. The Web-based tailoring engine would then use this information to present corresponding tailored messages incorporated with evidence-based informational content about vaccination to each user. The process by which we developed and evaluated the values tailoring and message framing strategies is described in the following sections.

\section{Values Survey}

We first conducted a survey among KPCO mothers to identify which values were important for vaccination and whether values 
may have an impact beyond tailoring on beliefs. Described elsewhere [24], these data suggested that values had an influence on vaccination behavior that was separate but additive to the influence from beliefs and should therefore be included as a tailoring target. In this paper, we report our exploratory assessment of the associations between values and different beliefs and concerns as posited that some values would be better matched than others to some vaccination beliefs. Owing to time constraints of the study, we used email to recruit a convenience sample of mothers of children aged between 1 and 4 years who were currently enrolled at KPCO. Surveys were completed on the Web using REDCap (Research Electronic Data Capture, Nashville, TN, USA) and SurveyGizmo (SurveyGizmo, Boulder, CO, USA) survey systems. A US \$30 incentive was provided. In addition to vaccination values, the survey also included measures of vaccination attitudes and beliefs and a global values measurement scale called the Schwartz Theory of Basic Human Values [21] to ascertain how values and attitudes relate to one another. Specific survey measures that were included are described in the following sections.

\section{Vaccine Values}

Parental values pertaining to childhood vaccination decisions (vaccine values) were measured using a scale the study team developed and assessed during this survey, called the Parental Vaccine Value Scale (PVVS). The PVVS is a 20-item scale that assesses 6 domains of values related to vaccination: security-disease prevention (valuing protecting one's children from the harm of infectious disease; Cronbach alpha=.74), security-vaccine risk (valuing protecting one's children from perceived harm of vaccines; $\alpha=.73$ ), universalism (valuing protecting one's community as a whole from the harm of infectious disease; $\alpha=.86$ ), self-direction (valuing the process of gathering information to make an informed decision; $\alpha=.66$ ), conformity (valuing the recommendations of experts and authority; $\alpha=.62$ ), and tradition (valuing following the established norm in one's religion or family; $\alpha=.79$ ). These 6 domains were created to reflect a subset of global values in the Schwartz Theory of Basic Human Values, which was also examined during the survey $[21,41,42]$. The development and validation of the PVVS, including factor structure and alignment with the Schwartz global values, is described in a separate publication [24]. On the basis of the results of this analysis, the PVVS was chosen in favor of the Schwartz Theory of Basic Human Values scale as the tailoring target in the intervention. Values were assessed with a 4-point Likert scale that ranged from strongly agree to strongly disagree.

\section{Vaccine Beliefs and Concerns}

Beliefs and concerns about childhood vaccination were measured using a 10-item scale developed in a previous study [39], with three additional questions later added by the study team for the purposes of the project. A 5-point Likert scale (strongly agree to strongly disagree) was used to assess the responses.

\section{Intention to Vaccinate}

In all, 2 items that assessed mothers' intention to vaccinate their newborn during the first year of life were used for message tailoring. These were based on performance of similar questions in a past study [39]. One item assessed how many of the 8 vaccines in the infant series a participant planned to have their infant receive with the following options: none of the vaccines, some of the vaccines, and all of the vaccines. The second item assessed when the mother intended to have their infant vaccinated with the following response options: all on-time as recommended by my baby's doctor and all or some later than my baby's doctor recommends (often referred to as a delayed scheduled or an alternative schedule). Combining these 2 items, mothers were categorized into 3 groups: refuses all vaccines, uses an alternative schedule (receives some or all vaccines but does not follow recommended timing), and full vaccine acceptor. The first 2 of these categories were grouped together to define vaccine-hesitant mothers when assessing vaccination outcomes at the end of the trial.

\section{Analysis of Survey Data}

From the survey data, we computed Spearman correlations between PVVS domain scores and the belief items. These results, combined with subsequent research team input, qualitative data, and expert opinion, were used to determine which values were associated with each specific belief or concern about vaccination and would therefore be included in the tailoring algorithm.

\section{Interviews Exploring Message Framing and Values Incorporation}

We considered several message framing options for the intervention messages. Using general information marketing approaches [43-45], we evaluated the potential usefulness of combining message framing with values framing approaches for the intervention messages. We considered the following approaches: 1) only values tailoring; (2) 1-sided messages, which present information about vaccines without acknowledgement of antivaccine arguments or negative aspects of vaccination [44]; (3) nonrefutational two-sided messages, which present both provaccination information and potential negatives (such as side effects) to the same extent (50\% of information is pro and $50 \%$ is con) [44]; (4) push messages, which directly refute myths about vaccines and adopt a directive tone; and (5) pull messages, which provide information and invite the reader into a conversation or discussion about the topic while trying to encourage the desired action or behavior and encourage central processing or personal engagement in the content $[37,43,45]$.

These various framing options were tested, along with the impact of different values and beliefs combinations, using structured interviews with 7 new mothers. The interviews took place between April and July 2015. Mothers were recruited from participants who took the survey described above and were eligible if they indicated in the survey an interest in providing feedback about vaccine messages, their child was at least 30 days undervaccinated as indicated in the medical record, and the mother had endorsed at least one of the six vaccine values domains in their survey (however, the messages tested were not necessarily matched to mothers' most highly affirmed values, though this was attempted when possible). Potential participants were recruited using email and phone outreach. The interviews 
focused on assessing the participant's acceptability of the messages and preferences between different formats. The interviews continued until saturation was reached. The participants received a US $\$ 50$ gift card for their time.

We designed the architecture of the intervention based on the concept of a home page consisting of different tiles representing different vaccination issues (Figure 3 ) as a central navigation point. We planned to have the most highly tailored information for the intervention presented in 3 Just for You tiles located prominently on the top of home page (Figure 3). These tiles represented the mothers' three most pressing concerns or questions regarding vaccination. The intent of these tiles was to (1) facilitate the ease with which each parent could locate the content of highest interest, (2) increase mothers' receptivity to information about their vaccine concern(s), (3) succinctly and credibly summarize key information about the concern, and (4) provide interested mothers with access to additional information about their concern. In the message framing interviews, 4 mothers were presented with up to 9 messages, which addressed as many as three featured concerns in the Just for You tiles. These messages included up to three messages tailored to value domains and up to three messages with different framing options (ie, a $3 \times 3$ factorial design; see Table 1 for examples of values tailoring messages). After viewing all the messages for an area of concern, mothers ranked the messages in order of their preferences and described their reasoning around their rankings. When a mother's top choices aligned with their measured values, we considered the message a candidate for the final intervention. When mothers' measured values and top ranked messages conflicted, we revised the messages to better align with the reasons that the mothers provided in the message framing interviews. Revised messages were further tested in subsequent interviews using the same interview format but with 3 different mothers.

Figure 3. Architecture of the Final Tailored Intervention - Vaccines and Your Baby Home Page.
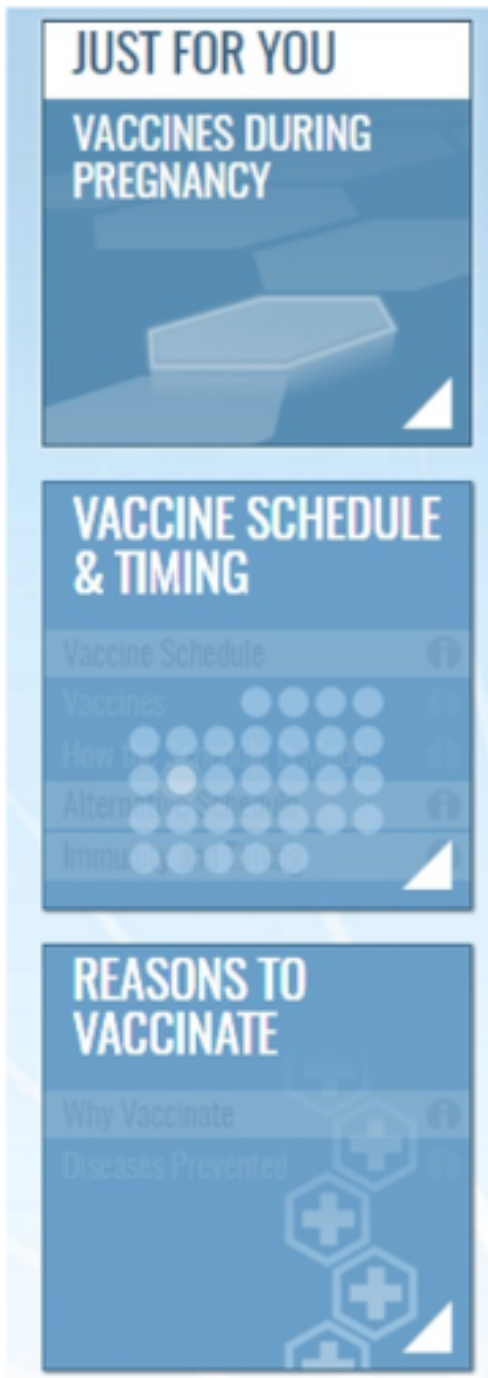
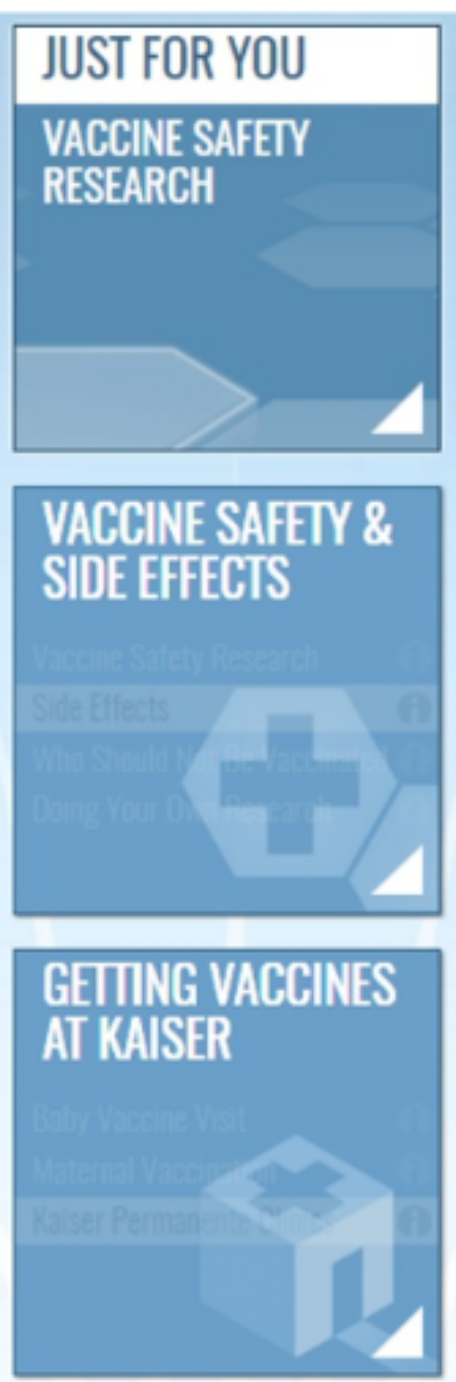
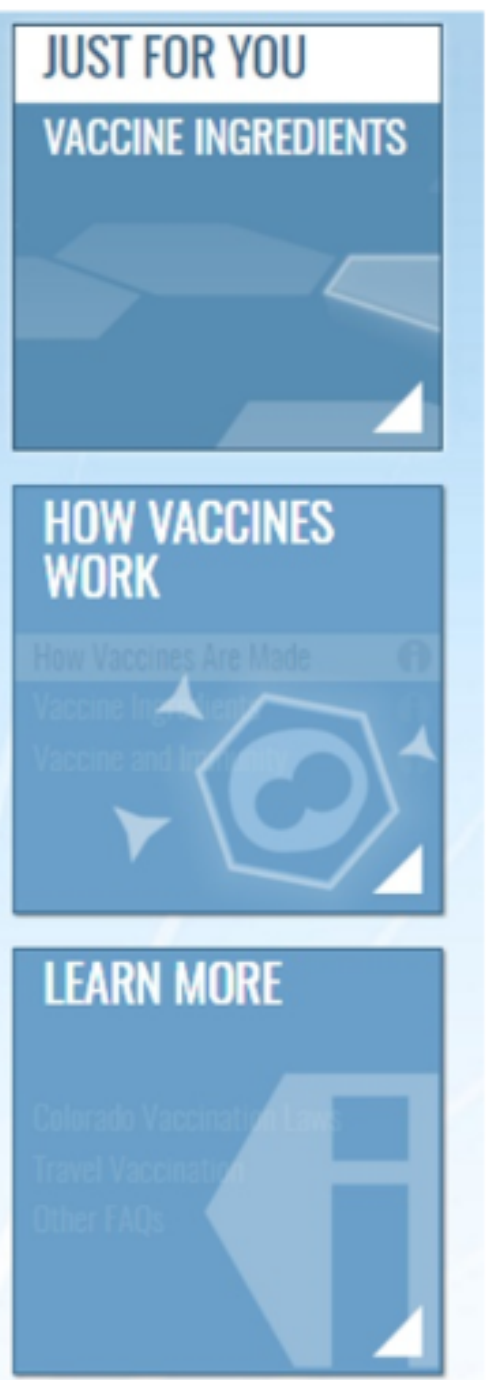
Table 1. Examples of values-tailored messages. (Vaccines and Your Baby: Tailored Messages.)

\begin{tabular}{|c|c|c|}
\hline \multirow[t]{2}{*}{ Value } & \multicolumn{2}{|l|}{ Topics } \\
\hline & Alternative/delayed vaccine schedules message & $\begin{array}{l}\text { Doing your own research on vaccines } \\
\text { message }\end{array}$ \\
\hline $\begin{array}{l}\text { Security-dis- } \\
\text { ease prevention }\end{array}$ & $\begin{array}{l}\text { Like many parents, your main goal is to keep your child healthy. The last thing you want } \\
\text { is for your child to get an illness you could have prevented with a simple vaccine. }\end{array}$ & $\begin{array}{l}\text { You're the kind of person who will do } \\
\text { everything she can to protect her baby } \\
\text { from illnesses. }\end{array}$ \\
\hline $\begin{array}{l}\text { Security-vac- } \\
\text { cine risk }\end{array}$ & $\begin{array}{l}\text { That's a lot of needles (and a lot of tears)! You want to protect your child. But with so } \\
\text { many vaccines at once, you're concerned about exposing him/her to too many unnatural } \\
\text { ingredients all at once. }\end{array}$ & $\begin{array}{l}\text { You're the kind of person who will do } \\
\text { everything she can to protect her baby } \\
\text { from pain or unnecessary medicines. }\end{array}$ \\
\hline
\end{tabular}

\section{Tailoring Components}

\section{Tailoring on Beliefs, Concerns, Hesitancy, and Demographics}

Consistent with many previous tailored interventions and the $\mathrm{TPB}$, we planned a priori to incorporate tailoring based on mothers' beliefs and concerns, intention to vaccinate, and demographic characteristics, as described in the following sections.

\section{Belief Tailoring}

Of the 13 vaccination concerns assessed in the preintervention survey, those receiving an average score of 3 or less (some were composite measures, possible range of 1-5, with lower values corresponding to increasing antivaccine views) were considered a qualifying concern that could potentially be tailored on. This value was chosen as the cutoff for categorizing a concern as qualifying, given that it would capture participants with less-than-positive (ie, neutral or negative) beliefs about the topic. If more than three concerns met this threshold, mothers were asked to select their top three concerns that formed the basis of the Just for You tiles. If only three concerns were identified, they became the 3 Just for You tiles. Concerns beyond the top 3 selected by the mother were highlighted in the website's base content (see Figure 3). If there were less than three concerns, up to 3 default Just for You tiles were featured in priority order: The Vaccine Schedule, Baby Vaccine Visit, and Kaiser Permanente Clinics. These topics were chosen as they were felt to have the least potential for raising new concerns among mothers without at least three concerns. For mothers with no vaccination concerns, Just for You content was tailored (first tile only) by providing positive reinforcement about their decision to vaccinate. The vaccine schedule tile was tailored based on the child's age at the time the website was being viewed.

\section{Intention Tailoring}

We tailored content on vaccination intention based on responses to the 2 vaccine intention questions described earlier (how many of the 8 vaccines in the infant series a participant planned to have their infant receive and when the mother intended to have their infant vaccinated). These responses were combined, and mothers were categorized into 1 of 3 mutually exclusive groups for tailoring (as opposed to only 2 groups for the planned final vaccination analysis of the trial) that was incorporated into the content of the Just for You tiles: refuses all vaccines, uses an alternative schedule (receives some or all vaccines but does not follow recommended timing), and full vaccine acceptor.

\section{Demographic Tailoring}

Tailoring on personal characteristics, including the mother's pregnancy status and child's nickname, was incorporated throughout the website content.

\section{Results}

\section{Developing Informational Content}

In total, there were 17 general topic areas that the intervention addressed, divided into 41 specific content areas (Table 2). These 41 content areas were further grouped into 6 broad categories corresponding to the 6 standard tiles (ie, not the Just for You tiles) presented on the intervention's home page (Figure 3 ). Furthermore, 4 of the 41 content areas were newly developed for the intervention. The remaining 37 were adapted from the previous intervention [39]. In addition, three newly developed, interactive infographics were also included on the following topics: herd immunity (included under the Community Benefits topic), antigen counts in the past and current vaccines (included under the Vaccine Ingredient Types topic), and disease rates before and after vaccines (included under the Risk of Diseases topic). Content areas included in the 6 home page tiles were identical in the tailored and untailored websites. 
Table 2. Topic areas (general tops: $n=17$; specific topics: $n=41$ ) covered in the intervention and corresponding tile on the home page ( $n=6$ ).

\begin{tabular}{ll}
\hline General topic area of interest and specific topics covered within each $\quad$ Name of corresponding tile on home pag
\end{tabular}

\section{Vaccine schedule}

Recommended vaccine schedule

Vaccines

Safety of the schedule

How the schedule is made

Importance of vaccine timing

\section{Alternative schedules}

Reasons why we cannot recommend an alternative schedule

\section{Immunity and timing}

Baby's developing immune system

Parents' main concerns about baby vaccines

\section{Vaccine safety research}

How vaccine studies are done

Vaccine Adverse Event Reporting System, Vaccine Safety Datalink, Vaccine Injury Compensation Program-How vaccine side effects are reported

Doing your own research ${ }^{\mathrm{a}}$

\section{Vaccine side effects}

Mild/common side effects

Serious/rare side effects

Illnesses/conditions not currently linked to vaccines

Who should be vaccinated?

\section{How vaccines are made}

Vaccine production

How Pharma works

Kaiser and Pharma

\section{Vaccine ingredients}

Why some ingredients are needed for vaccine production

Vaccine ingredient types

Individual ingredients

\section{Vaccines and immunity}

Natural versus vaccine immunity

\section{Why vaccinate}

Community benefits of vaccination

Risk of diseases ${ }^{\mathrm{a}}$

Worldwide risk of diseases ${ }^{\mathrm{a}}$

Current outbreaks (measles and pertussis) ${ }^{\mathrm{a}}$

\section{Diseases prevented}

Vaccine-preventable diseases and vaccines given at Kaiser Permanente Colorado

\section{The baby vaccine visit}

Before the vaccine visit

During the vaccine visit
Vaccine schedule and timing

Vaccine schedule and timing

Vaccine schedule and timing

Vaccine schedule and timing

Vaccine schedule and timing

Vaccine schedule and timing

Vaccine schedule and timing

Vaccine schedule and timing

Vaccine safety and side effects

Vaccine safety and side effects

Vaccine safety and side effects

Vaccine safety and side effects

Vaccine safety and side effects

Vaccine safety and side effects

Vaccine safety and side effects

How vaccines work

How vaccines work

How vaccines work

How vaccines work

How vaccines work

How vaccines work

How vaccines work

Reasons to vaccinate

Reasons to vaccinate

Reasons to vaccinate

Reasons to vaccinate

Reasons to vaccinate

Getting vaccines at Kaiser

Getting vaccines at Kaiser 


\begin{tabular}{|c|c|}
\hline General topic area of interest and specific topics covered within each & Name of corresponding tile on home page \\
\hline After the vaccine visit & Getting vaccines at Kaiser \\
\hline \multicolumn{2}{|l|}{ Maternal vaccination } \\
\hline Vaccines in pregnancy & Getting vaccines at Kaiser \\
\hline Safety of vaccines in pregnancy & Getting vaccines at Kaiser \\
\hline \multicolumn{2}{|l|}{ Kaiser Permanente clinics } \\
\hline Transportation to Kaiser clinics & Getting vaccines at Kaiser \\
\hline Bus routes & Getting vaccines at Kaiser \\
\hline Kaiser clinic hours & Getting vaccines at Kaiser \\
\hline \multicolumn{2}{|l|}{ Colorado vaccine laws } \\
\hline Colorado vaccine laws & Learn more \\
\hline Colorado vaccine exemptions & Learn more \\
\hline \multicolumn{2}{|l|}{ Travel vaccination } \\
\hline Kaiser travel clinic & Learn more \\
\hline Vaccines and traveling abroad & Learn more \\
\hline \multicolumn{2}{|l|}{ Adolescent vaccination } \\
\hline Adolescent vaccination & Learn more \\
\hline \multicolumn{2}{|l|}{ Other FAQs ${ }^{b}$} \\
\hline Other FAQs & Learn more \\
\hline
\end{tabular}

${ }^{\mathrm{a}}$ Newly developed for the intervention.

${ }^{b}$ FAQ: frequently asked question.

\section{Assessing and Finalizing the Architecture of the Intervention}

\section{Design Interviews}

Participants included 3, white, non-Hispanic mothers of children aged between 1 and 4 years. All the mothers preferred design features that appeared to be associated with the health system. This included color palettes with blue and green and logos with clean lines, similar to KPCO website pages. On the basis of the user feedback, we selected a tailored (and matching untailored) website design that used an interactive tile-based homepage (Figure 3), menu navigation in multiple locations, layering of information using accordion style grouping, and pop-up information in select locations.

\section{Usability Testing and Satisfaction Survey Results}

Of 271 mothers contacted for usability and satisfaction testing sessions, we scheduled a convenience sample of the first 6 respondents for usability testing interviews. Mothers who completed the interviews $(n=5)$ were all female, white, non-Hispanic, with at least some college education, and had income ranging from US $\$ 50,000$ to US $\$ 90,000$ or more. On the basis of their input, changes to the prototype architecture included adding submenus to the main page, using words in place of images to assist in identifying the schedule toggle feature, adding new links to content pages, and automatically closing content accordions to assist in reading and navigating long content areas. All but 1 user had SUS scores above the passable score of 70 (range 65-97.5), suggesting that the usability of the site was acceptable. Owing to the similarity in responses among mothers, additional interviews beyond these 5 were not undertaken.

\section{Description of the Architecture of the Final Tailored Intervention}

In the final intervention, mothers are first directed to an onboarding page designed to engage the participant in the website so that they are inclined to continue to view the content. It includes a welcome message, references the participant's intention to vaccinate, explains the intent of the website and that the information presented will be tailored based on their survey responses, and visually depicts where they can find the tailored content.

Following this, mothers are taken to the main home page of the intervention (Figure 3). On this page, the bulk of message tailoring is received via the 3 featured Just for You tiles. Any additional topics of concern beyond the top three concerns are highlighted within the 6 interactive tiles on this page. All the tiles lead to additional content that is tailored on the participant's personal characteristics.

\section{Iterative Development of Tailored Messages Incorporating Values and Framing}

\section{Values Survey Results}

Table 3 shows the results of correlations between belief items and values. These results informed team discussions about which values might be most appropriate for framing each concern topic. A combination of these data, research team consensus, 
and results from the parent interviews (described in the following sections) were used to determine which values were available as tailoring targets for each of the vaccine beliefs and concerns.

Table 3. Correlations between values and beliefs corresponding to website topic areas. Correlations (r) are Spearman correlation coefficients.

\begin{tabular}{|c|c|c|c|c|c|c|}
\hline \multirow[t]{2}{*}{ Beliefs $^{\mathrm{a}}$} & \multicolumn{3}{|c|}{ Associations with provaccine beliefs } & \multicolumn{3}{|c|}{ Associations with antivaccine beliefs } \\
\hline & Universalism, $\mathrm{r}$ & Conformity, $\mathrm{r}$ & $\begin{array}{l}\text { Security (disease preven- } \\
\text { tion), } \mathrm{r}\end{array}$ & $\begin{array}{l}\text { Security (vaccine } \\
\text { risk), } \mathrm{r}\end{array}$ & Tradition, $r$ & Self-direction, $r$ \\
\hline Enough research & $-0.249^{\mathrm{b}}$ & $-0.202^{\mathrm{c}}$ & -0.126 & $0.339^{\mathrm{d}}$ & $0.247^{\mathrm{b}}$ & $0.261^{b}$ \\
\hline Disease risk and benefit & $-0.423^{\mathrm{d}}$ & $-0.257^{\mathrm{b}}$ & $-0.311^{\mathrm{b}}$ & $0.202^{c}$ & $0.199^{\mathrm{c}}$ & 0.080 \\
\hline Too many, too soon & -0.139 & $-0.364^{\mathrm{d}}$ & $-0.199^{\mathrm{c}}$ & $0.411^{\mathrm{d}}$ & $0.257^{\mathrm{b}}$ & $0.365^{\mathrm{d}}$ \\
\hline Natural immunity & $-0.315^{\mathrm{b}}$ & $-0.226^{\mathrm{c}}$ & $-0.204^{\mathrm{c}}$ & $0.259^{\mathrm{b}}$ & $0.324^{\mathrm{d}}$ & 0.106 \\
\hline Vaccine safety & -0.123 & -0.173 & -0.078 & $0.352^{\mathrm{d}}$ & $0.360^{\mathrm{d}}$ & 0.180 \\
\hline Do own research & -0.173 & $-0.180^{\mathrm{c}}$ & $-0.221^{\mathrm{c}}$ & $0.249^{b}$ & 0.178 & $0.505^{\mathrm{d}}$ \\
\hline Vaccine ingredients & $-0.251^{\mathrm{b}}$ & $-0.347^{\mathrm{d}}$ & -0.117 & $0.239^{b}$ & $0.240^{\mathrm{b}}$ & 0.156 \\
\hline Autism & $-0.205^{\mathrm{c}}$ & $-0.231^{\mathrm{c}}$ & -0.092 & $0.338^{\mathrm{d}}$ & $0.358^{\mathrm{d}}$ & 0.164 \\
\hline $\begin{array}{l}\text { Vaccine risk versus } \\
\text { benefit }\end{array}$ & $-0.186^{\mathrm{c}}$ & -0.115 & $-0.343^{\mathrm{d}}$ & $0.474^{\mathrm{d}}$ & $0.344^{\mathrm{d}}$ & $0.333^{\mathrm{d}}$ \\
\hline Combined risk/benefit & $-0.319^{\mathrm{d}}$ & -0.176 & $-0.351^{\mathrm{d}}$ & $0.412^{\mathrm{d}}$ & $0.301^{\mathrm{d}}$ & $0.257^{\mathrm{d}}$ \\
\hline
\end{tabular}

${ }^{\mathrm{a}}$ Three additional vaccine topics were added based on expert feedback after this analysis was completed for a total of 13 belief topics.

${ }^{\mathrm{b}} \mathrm{P}<.01$

$\mathbf{c}_{\mathrm{P}<.05 \text {. }}$

$\mathbf{d}_{\mathrm{P}<.001 .}$

\section{Interviews Exploring Message Framing and Values Incorporation}

Of the 39 mothers contacted for interviews, 7 participated. All of them were mothers of children aged between 1 and 2 years.

Across topic areas, qualitative interviews assessing the general approach for framing messages revealed that two-sided messages were preferred by mothers compared with 1-sided, push, or pull messages. The two-sided message on side effects, presented as a table, was particularly well received. All mothers responded favorably to the intervention layout and reported that presenting both mild and rare side effects seemed honest. On the topic of alternative schedules (delaying or skipping certain vaccines), mothers generally $(n=5)$ preferred the two-sided messages, largely because of the detail provided. The 2 mothers who followed an alternative schedule disliked the black-and-white messaging against this practice, believing this approach could sway mothers away from vaccinating at all. Finally, when 1and two-sided messages were presented without values framing, mothers often $(n=5)$ mentioned that values framing would improve the likeability of the message.

Among the values-framed messages, most $(n=4)$ mothers preferred statements from self-direction values-tailored versions that acknowledged their right to choose. Mothers also responded favorably to the tailored versions of security that specifically addressed mothers' motivation for keeping their child safe. Values-framed messages around conformity were the least preferred, with only 1 mother in the sample endorsing this value strongly. Values were not favored by any of the mothers for messages on the topic of side effects. These findings guided the team to adopt a two-sided messaging approach that incorporated values tailoring for topics of concern identified for mothers.

\section{Tailoring Components}

\section{Final Values Tailoring Algorithm}

To build the final values tailoring algorithm, we used the results from the survey and interviews to identify the values or set of values that appeared impactful and relevant for each belief item assessed (Table 4). Any values exceeding the threshold of a 2.5 score (a score well above the neutral value of 2 on the 4-point scale used to assess values) that were also deemed impactful for that belief based on the survey and interview results were considered relevant values. We built an algorithm to randomly select one of these values and incorporate it into the corresponding Just for You tile. If the value has already been used in a previous topic area, another available value is selected at random from the available pool of values for that topic. If no additional values remain, all previously available values are made available for random selection. If the participant has no values that meet the threshold, a message without values framing for that vaccination concern is displayed. Values are generally incorporated into these tiles as wrap-around introductory sentences allowing for the core informational content on that belief to remain similar for each user. The same home page structure consisting of 9 tiles (Figure 2) was used for each retailoring. 
Table 4. Values available for tailoring according to topic areas of the Just for You Tiles.

\begin{tabular}{|c|c|c|c|c|c|c|}
\hline \multirow{2}{*}{$\begin{array}{l}\text { Title of the Just for You tile (content of corresponding } \\
\text { general or specific topic areas potentially linked to tile) }\end{array}$} & \multicolumn{6}{|c|}{ Applicable values } \\
\hline & $\begin{array}{l}\text { Security—dis- } \\
\text { ease risk }\end{array}$ & $\begin{array}{l}\text { Security-vac- } \\
\text { cine risk }\end{array}$ & $\begin{array}{l}\text { Self-direc- } \\
\text { tion }\end{array}$ & Conformity & Universalism & Tradition \\
\hline Vaccine Safety Research (vaccine safety research) & $x^{b}$ & $\mathrm{x}$ & $\mathrm{x}$ & $\mathrm{x}$ & $-^{\mathrm{c}}$ & - \\
\hline $\begin{array}{l}\text { Vaccine-Preventable Diseases (why vaccinate and dis- } \\
\text { eases prevented) }\end{array}$ & $\mathrm{x}$ & $\mathrm{x}$ & $\mathrm{x}$ & $\mathrm{x}$ & $\mathrm{x}$ & - \\
\hline $\begin{array}{l}\text { Number and Timing of Vaccines (how the schedule is } \\
\text { made, reasons why we cannot recommend an alternative } \\
\text { schedule, immunity and timing, and vaccines and immu- } \\
\text { nity) }\end{array}$ & $\mathrm{x}$ & $\mathrm{x}$ & $\mathrm{x}$ & $\mathrm{x}$ & - & - \\
\hline $\begin{array}{l}\text { Vaccine Ingredients (vaccine safety research and vaccine } \\
\text { ingredients) }\end{array}$ & $\mathrm{x}$ & $\mathrm{x}$ & $\mathrm{x}$ & $\mathrm{x}$ & - & - \\
\hline $\begin{array}{l}\text { Vaccine Side Effects (vaccine safety research and vaccine } \\
\text { side effects) }\end{array}$ & $\mathrm{x}$ & $\mathrm{x}$ & $\mathrm{x}$ & - & - & - \\
\hline $\begin{array}{l}\text { Doing Your Own Research on Vaccines (vaccine safety } \\
\text { research and doing your own research) }\end{array}$ & $\mathrm{x}$ & $\mathrm{x}$ & $\mathrm{x}$ & $\mathrm{x}$ & $\mathrm{x}$ & - \\
\hline $\begin{array}{l}\text { The Immune System and Vaccines (immunity and timing, } \\
\text { vaccines and immunity, and why vaccinate) }\end{array}$ & $\mathrm{x}$ & $\mathrm{x}$ & - & $\mathrm{x}$ & $\mathrm{x}$ & - \\
\hline $\begin{array}{l}\text { Vaccines and Autism (side effects and vaccine ingredi- } \\
\text { ents) }\end{array}$ & $\mathrm{x}$ & $\mathrm{x}$ & $\mathrm{x}$ & - & - & - \\
\hline $\begin{array}{l}\text { Vaccination Risks and Benefits (vaccine side effects, who } \\
\text { should not be vaccinated, and why vaccinate) }\end{array}$ & $\mathrm{x}$ & $\mathrm{x}$ & $\mathrm{x}$ & - & $\mathrm{x}$ & - \\
\hline Vaccines During Pregnancy (maternal vaccination) & $\mathrm{x}$ & $\mathrm{x}$ & $\mathrm{x}$ & $\mathrm{x}$ & - & - \\
\hline $\begin{array}{l}\text { The Role of Pharmaceutical Companies (how vaccines } \\
\text { are made) }\end{array}$ & - & $\mathrm{x}$ & $\mathrm{x}$ & - & - & - \\
\hline $\begin{array}{l}\text { Alternative/Delayed Vaccine Schedules (reasons why we } \\
\text { cannot recommend an alternative schedule, immunity, } \\
\text { and timing) }\end{array}$ & $\mathrm{x}$ & $\mathrm{x}$ & $\mathrm{x}$ & & & - \\
\hline $\begin{array}{l}\text { Tips for Vaccinating at Kaiser (recommended vaccine } \\
\text { schedule, baby vaccine Visit, and Kaiser Permanente } \\
\text { clinics) }\end{array}$ & $\begin{array}{l}\text { No values tai- } \\
\text { loring }\end{array}$ & $\begin{array}{l}\text { No values tai- } \\
\text { loring }\end{array}$ & $\begin{array}{l}\text { No values } \\
\text { tailoring }\end{array}$ & $\begin{array}{l}\text { No values } \\
\text { tailoring }\end{array}$ & $\begin{array}{l}\text { No values tai- } \\
\text { loring }\end{array}$ & $\begin{array}{l}\text { No values } \\
\text { tailoring }\end{array}$ \\
\hline
\end{tabular}

${ }^{\mathrm{a}}$ Multiple General or Specific Topics related to several of the Just for You tiles. These tiles could include information on 1 or more general or specific topics depending on the user's input.

${ }^{\mathrm{b}} \mathrm{An}$ ' $\mathrm{x}$ ' in the table denotes that a given value is available to incorporate into the Just for You tile content.

${ }^{\mathrm{c}} \mathrm{A}$ ' - ' in the table denotes that the given value is not available to incorporate into the Just for You tile content.

\section{Discussion}

\section{Principal Findings}

In this paper, we described in detail how we developed a Web-based, tailored messaging intervention to address maternal vaccine hesitancy using an iterative development process and a mixed method approach. This intervention, which was developed using validated health behavior theories, is expected to be effective, engaging, and easy to use based on end-user feedback and pilot testing. Ultimately, the intervention included common tailoring elements such as demographics and beliefs as well as a novel tailoring target, personal values. In addition to examining tailoring targets, we also used user feedback to assess the potential impact of different message framing strategies in combination with the tailored elements. This was necessary as there is ongoing debate about the optimal messages framing strategy with regard to vaccine hesitancy and immunization [46-51].

\section{Application of Theory}

Of the 4 health behavior theories and techniques used to inform the development of the intervention, the TPB has the most evidence for its applicability to vaccine decision making. Owing to this, we planned a priori to incorporate elements from this theory into our intervention and therefore did not focus any of our data collection described in this paper on these elements. Specifically, the intervention was tailored based on TPB constructs of attitudes, and when possible, elements of social norms were incorporated into the messages. The other remaining theories and techniques - the VAB theory and elements of persuasive messaging and MI-did not have a strong evidence base with regard to vaccine decision making at the time the intervention was being developed. However, the data collected in this study suggest that each is relevant to the vaccination 
decision. Specifically, results from our quantitative survey of values and from the qualitative interviews on message framing and values tailoring both suggest that certain values are more important than others with regard to vaccine decision making, and that some values are better incorporated with certain beliefs than others. A subsequent study done by our group examining the role that values play in mothers' vaccine decision making further supports this notion [24]. Support for incorporating techniques from persuasive messaging into the intervention was also derived from the qualitative message framing and values tailoring interviews. The respondents clearly indicated that vaccine-hesitant parents strongly preferred messages that were perceived as balanced, where both positive and negative information (ie, two-sided messages) about vaccine safety or benefits was presented as these messages were deemed more trustworthy than messages that only conveyed one side of these issues. This point supports the concept in persuasive messaging that messages deemed as trustworthy are more likely to be reflected upon and more persuasive. Also supporting the importance of persuasive messaging was the finding from the message framing and values tailoring interviews that showed that messages tailored to the user were deemed more personally relevant. The design feedback interviews showing that mothers uniformly liked intervention architecture that clearly allowed a user to choose the specific information to view also support this concept. Evidence from our study for incorporating techniques from MI into the intervention is somewhat indirect in that mothers participating in the message framing and values interviews who were following an alternative vaccination schedule did not like black-and-white messages that argued against this practice. Such messages could be considered to counter the MI tenet of rolling with resistance. Another important MI tenet is that of using intrinsic motivation to effect behavior change. Evidence in support of this concept also comes from the message framing and values tailoring interviews showing that mothers generally preferred values-tailored messages to those not tailored to values. Further work by our group and others that occurred after our intervention was developed lends additional support to the important role that MI likely plays in motivating parents to vaccinate $[52,53]$.

\section{Future Work}

The next step in our study is to assess the efficacy of this intervention in a randomized controlled trial of KPCO expectant and new mothers. This trial (ClinicalTrials.gov protocol number NCT02665013) will examine the relative effect of the tailored versus untailored websites for their effect on timely infant vaccine utilization during the first 15 months of life. In addition, a number of secondary outcomes will also be assessed including whether the intervention modified maternal vaccination beliefs and concerns or vaccine hesitancy, and how any changes in these outcomes relate to vaccination values. This will allow for a more thorough investigation of the VAB model used as a basis for this study.

\section{Potential Importance of Values}

Tailoring on values represents a novel, and potentially important, innovation incorporated into this intervention. A large body of literature in the social sciences provides compelling evidence that aligning educational messages with personal values can make information more salient and actionable [54-57]. The somewhat more limited number of studies examining values tailoring in the realm of health behavior change specifically further support this concept [58-61]. Recently, researchers interested in improving immunization delivery have begun to recognize the potential role of values tailoring in promoting vaccination [23,27,62]. Although there has been limited prior research examining the impact of values tailoring on vaccine acceptance, our own study [24] and that of others [63], which were completed after the study presented in this paper, have shown that differences in personal values are associated with variation in the acceptance of recommended vaccines. Taken together, values appear to be a potentially untapped tailoring resource that warrants further exploration. We believe results from the randomized controlled trial that will evaluate this intervention will add important and novel information to this growing body of research.

\section{Limitations}

This paper should be considered in the context of some important limitations. First, when developing the various aspects of the intervention, we generally received input from only a small number of mothers, most of whom were white and non-Hispanic. Maternal input was not designed to be comprehensive. Rather, we opted to obtain in-depth information from a handful of mothers to provide more nuanced insight for optimizing the intervention and making it more relevant to potential end users, and we generally solicited mothers' input until thematic saturation was reached (although we did not do a formal qualitative analysis). In addition, we focused much of the intervention development on the incorporation of values as a tailoring element, an approach to tailoring that is in need of further study. Moreover, although the study population providing input into the interventions' design was diverse with regard to demographic characteristic such as race, ethnicity, education, and income, the population was relatively homogeneous with regard to insurance status (all had access to care) and primary language (we only gathered data from English-speaking mothers) and did not include fathers. As such, any impact of the intervention found in the upcoming clinical trial may not be generalizable to other populations that differ in these respects, and the intervention may need further refinement based on these characteristics. In addition, there are several items that previous research has shown as potentially important variables in the vaccination decision that could represent potential tailoring variables (education level, exposure to scientific controversy, degree of social networking, etc) $[64,65]$ and were not included in our intervention. This was a purposeful decision to be able to isolate any potential impacts of values tailoring on vaccine uptake. However, these variables may need to be considered as tailoring targets in future iterations of the intervention. A notable strength of the project was the use of multiple methods to collect data (qualitative and quantitative), which may increase the validity of our findings.

\section{Conclusions}

We used both qualitative and quantitative approaches and significant end-user input to develop a Web-based, 
theory-driven, tailored messaging intervention designed to address maternal vaccine hesitancy and subsequently improve uptake of infant vaccines. Our results suggest that elements from each of the 4 models and techniques incorporated into our conceptual model for the intervention were important. Specifically, results of qualitative user design and message framing interviews supported using several techniques from persuasive messaging and $\mathrm{MI}$ in the intervention. A quantitative survey on parents' vaccination values, combined with qualitative data from the message framing interviews, supported the importance of the VAB model as a foundation of the intervention and supported the idea of using values as a novel tailoring variable. Elements from the TPB were not assessed directly in this study as there was already an evidence base supporting the importance of this theory in parents' vaccine decision making. By incorporating elements from these four theories and techniques into the intervention, we believe it will be highly effective in changing mothers' vaccination attitudes and behaviors. This hypothesis will be tested in a subsequent randomized controlled trial.

\section{Acknowledgments}

This project was funded by the National Institutes of Health Eunice Kennedy Shriver National Institute of Child Health and Human Development (R01 HD079457).

\section{Conflicts of Interest}

AD serves on the advisory boards for Merck, Sanofi, and Pfizer and has worked as a consultant for Pfizer. None of these companies played a role in this research, and she does not receive research funding from these groups. All other authors have no conflicts to declare.

\section{References}

1. Roush SW, Murphy TV, Vaccine-Preventable Disease Table Working Group. Historical comparisons of morbidity and mortality for vaccine-preventable diseases in the United States. JAMA 2007 Nov 14;298(18):2155-2163. [doi: 10.1001/jama.298.18.2155] [Medline: 18000199]

2. Omer SB, Pan WK, Halsey NA, Stokley S, Moulton LH, Navar AM, et al. Nonmedical exemptions to school immunization requirements: secular trends and association of state policies with pertussis incidence. JAMA 2006 Oct 11;296(14):1757-1763. [doi: 10.1001/jama.296.14.1757] [Medline: 17032989]

3. Glanz JM, Newcomer SR, Narwaney KJ, Hambidge SJ, Daley MF, Wagner NM, et al. A population-based cohort study of undervaccination in 8 managed care organizations across the United States. JAMA Pediatr 2013 Mar 01;167(3):274-281. [doi: 10.1001/jamapediatrics.2013.502] [Medline: 23338829]

4. Omer SB, Salmon DA, Orenstein WA, deHart MP, Halsey N. Vaccine refusal, mandatory immunization, and the risks of vaccine-preventable diseases. N Engl J Med 2009 May 7;360(19):1981-1988. [doi: 10.1056/NEJMsa0806477] [Medline: 19420367]

5. Siddiqui M, Salmon DA, Omer SB. Epidemiology of vaccine hesitancy in the United States. Hum Vaccin Immunother 2013 Dec;9(12):2643-2648 [FREE Full text] [doi: 10.4161/hv.27243] [Medline: 24247148]

6. Sundaram ME, Guterman LB, Omer SB. The true cost of measles outbreaks during the postelimination era. JAMA 2019 Mar 26;321(12):1155-1156. [doi: 10.1001/jama.2019.1506] [Medline: $\underline{\text { 30844023] }}$

7. Salmon DA, Dudley MZ, Glanz JM, Omer SB. Vaccine hesitancy: causes, consequences, and a call to action. Vaccine 2015 Nov 27;33(Suppl 4):D66-D71. [doi: 10.1016/j.vaccine.2015.09.035] [Medline: 26615171]

8. N/A. Assessing the State of Vaccine Confidence in the United States: Recommendations from the National Vaccine Advisory Committee: Approved by the National Vaccine Advisory Committee on June 9, 2015 [corrected]. Public Health Rep 2015;130(6):573-595 [FREE Full text] [doi: 10.1177/003335491513000606] [Medline: 26556929]

9. Dempsey AF, Wagner N, Narwaney K, Pyrzanowski J, Kwan BM, Kraus C, et al. 'Reducing Delays In Vaccination' (REDIVAC) trial: a protocol for a randomised controlled trial of a web-based, individually tailored, educational intervention to improve timeliness of infant vaccination. BMJ Open 2019 May 22;9(5):e027968 [FREE Full text] [doi:

10.1136/bmjopen-2018-027968] [Medline: 31122997]

10. Trivedi D. Cochrane review summary: face-to-face interventions for informing or educating parents about early childhood vaccination. Prim Health Care Res Dev 2014 Oct;15(4):339-341. [doi: 10.1017/S1463423614000322] [Medline: 25146256]

11. Sadaf A, Richards JL, Glanz J, Salmon DA, Omer SB. A systematic review of interventions for reducing parental vaccine refusal and vaccine hesitancy. Vaccine 2013 Sep 13;31(40):4293-4304. [doi: 10.1016/j.vaccine.2013.07.013] [Medline: 23859839]

12. Dubé E, Vivion M, MacDonald NE. Vaccine hesitancy, vaccine refusal and the anti-vaccine movement: influence, impact and implications. Expert Rev Vaccines 2015 Jan;14(1):99-117. [doi: 10.1586/14760584.2015.964212] [Medline: 25373435]

13. MacDonald NE. Vaccine hesitancy: definition, scope and determinants. Vaccine 2015 Aug 14;33(34):4161-4164 [FREE Full text] [doi: 10.1016/j.vaccine.2015.04.036] [Medline: 25896383]

14. MacDonald NE, Butler R, Dubé E. Addressing barriers to vaccine acceptance: an overview. Hum Vaccin Immunother 2018 Jan 02;14(1):218-224. [doi: 10.1080/21645515.2017.1394533] [Medline: 29048975] 
15. Hawkins RP, Kreuter M, Resnicow K, Fishbein M, Dijkstra A. Understanding tailoring in communicating about health. Health Educ Res 2008 Jun;23(3):454-466 [FREE Full text] [doi: 10.1093/her/cyn004] [Medline: $\underline{18349033]}$

16. Kreuter MW, Strecher VJ, Glassman B. One size does not fit all: the case for tailoring print materials. Ann Behav Med 1999;21(4):276-283. [Medline: 10721433]

17. Noar SM, Benac CN, Harris MS. Does tailoring matter? Meta-analytic review of tailored print health behavior change interventions. Psychol Bull 2007 Jul;133(4):673-693. [doi: 10.1037/0033-2909.133.4.673] [Medline: 17592961]

18. Stockard J, Carpenter G, Kahle LR. Continutity and change in values in midlife: testing the age stability hypothesis. Experimental Aging Research 2014 Mar 13;40(2):224-244. [doi: 10.1080/0361073x.2014.882215]

19. Rokeach M. The Nature Of Human Values. New York: Free Press; 1973.

20. Konty MA, Dunham CC. Differences in value and attitude change over the life course. Sociological Spectrum 2010 Jul 30;17(2):177-197. [doi: 10.1080/02732173.1997.9982159]

21. Schwartz S. Are there universal aspects in the structure and contents of human values? J Soc Issu 1994;50:19-45. [doi: 10.1111/j.1540-4560.1994.tb01196.x]

22. Bedford H, Attwell K, Danchin M, Marshall H, Corben P, Leask J. Vaccine hesitancy, refusal and access barriers: the need for clarity in terminology. Vaccine 2018 Oct 22;36(44):6556-6558. [doi: 10.1016/j.vaccine.2017.08.004] [Medline: 28830694]

23. Leask J, Macartney K. Parental decisions about vaccination: collective values are important. J Paediatr Child Health 2008 Oct;44(10):534-535. [doi: 10.1111/j.1440-1754.2008.01381.x] [Medline: 19012625]

24. Cataldi JR, Sevick C, Pyrzanowski J, Wagner N, Brewer SE, Narwaney KJ, et al. Addressing personal parental values in decisions about childhood vaccination: measure development. Vaccine 2019 Sep;37(38):5688-5697. [doi: 10.1016/j.vaccine.2019.08.009]

25. Dore RA, Stone ER, Buchanan CM. A social values analysis of parental decision making. J Psychol 2014;148(4):477-504. [doi: 10.1080/00223980.2013.808603] [Medline: 24946390]

26. Salmon DA, Omer SB. Individual freedoms versus collective responsibility: immunization decision-making in the face of occasionally competing values. Emerg Themes Epidemiol 2006 Sep 27;3:13 [FREE Full text] [doi: 10.1186/1742-7622-3-13] [Medline: 17005041$]$

27. Witteman HO. Addressing vaccine hesitancy with values. Pediatrics 2015 Aug;136(2):215-217 [FREE Full text] [doi: 10.1542/peds.2015-0949] [Medline: 26195531]

28. Witteman HO, Chipenda Dansokho S, Exe N, Dupuis A, Provencher T, Zikmund-Fisher BJ. Risk communication, values clarification, and vaccination decisions. Risk Anal 2015 Oct;35(10):1801-1819. [doi: 10.1111/risa.12418] [Medline: 25996456]

29. Glanz JM, Wagner NM, Narwaney KJ, Shoup JA, McClure DL, McCormick EV, et al. A mixed methods study of parental vaccine decision making and parent-provider trust. Acad Pediatr 2013;13(5):481-488 [FREE Full text] [doi: 10.1016/j.acap.2013.05.030] [Medline: 24011751]

30. Glanz JM, Kraus CR, Daley MF. Addressing parental vaccine concerns: engagement, balance, and timing. PLoS Biol 2015 Aug;13(8):e1002227 [FREE Full text] [doi: 10.1371/journal.pbio.1002227] [Medline: 26252770]

31. Dempsey AF, Wagner N, Narwaney K, Pyrzanowski J, Kwan BM, Kraus C, et al. 'Reducing Delays In Vaccination' (REDIVAC) trial: a protocol for a randomised controlled trial of a web-based, individually tailored, educational intervention to improve timeliness of infant vaccination. BMJ Open 2019 May 22;9(5):e027968 [FREE Full text] [doi:

10.1136/bmjopen-2018-027968] [Medline: 31122997]

32. Glanz JM, Wagner NM, Narwaney KJ, Kraus CR, Shoup JA, Xu S, et al. Web-based social media intervention to increase vaccine acceptance: a randomized controlled trial. Pediatrics 2017 Dec;140(6):- [FREE Full text] [doi: 10.1542/peds.2017-1117] [Medline: 29109107]

33. Ajzen I. The theory of planned behavior. Organizational Beh Hum Dec Process 1991 Dec;50(2):179-211. [doi: 10.1016/0749-5978(91)90020-t]

34. Ajzen I. Values, Attitudes, and Behavior. In: Methods, Theories, and Empirical Applications in the Social Sciences. Wiesbaden: VS Verlag für Sozialwissenschaften; 2012:33-38.

35. Miller W, Rollnick S. Motivational interviewing. New York: Guilford Press; 1991.

36. O'Keefe G. Persuasion: Theory and Research. Thousand Oaks: SAGE Publications; 2002.

37. Petty R, Cacioppo JT. The elaboration likelihood model of persuasion. Adv Exp Med Soc Psych 1986;19:123-205. [doi: 10.1016/s0065-2601(08)60214-2]

38. Gagneur A, Gosselin V, Dubé È. Motivational interviewing: a promising tool to address vaccine hesitancy. Vaccine 2018 Oct 22;36(44):6553-6555. [doi: 10.1016/j.vaccine.2017.10.049] [Medline: 30301523]

39. Shoup JA, Wagner NM, Kraus CR, Narwaney KJ, Goddard KS, Glanz JM. Development of an interactive social media tool for parents with concerns about vaccines. Health Educ Behav 2015 Jun;42(3):302-312. [doi: 10.1177/1090198114557129] [Medline: 25413375]

40. Jordan P, Thomas B, Lyall I. SUS: A quick and dirty usability scale. In: Usability Evaluation in Industry. Bristol, PA: Taylor \& Francis; 1996.

41. Schwartz SH, Boehnke K. Evaluating the structure of human values with confirmatory factor analysis. J Res Personality 2004 Jun;38(3):230-255. [doi: 10.1016/s0092-6566(03)00069-2] 
42. Schwartz SH. An overview of the Schwartz theory of basic values. Online Read Psychol Cult 2012;2(1):- [FREE Full text] [doi: 10.9707/2307-0919.1116]

43. Olver J, Farris PW. Push and pull: a one-two punch for packaged products. MIT Sloan Manag Rev 1989;31(1):53-61.

44. O'Keefe DJ. How to handle opposing arguments in persuasive messages: a meta-analytic review of the effects of one-sided and two-sided messages. AICA 2016 May 18;22(1):209-249. [doi: 10.1080/23808985.1999.11678963]

45. Robertson T. Chron. 2019. Difference between push \& pull marketing URL: https://smallbusiness.chron.com/ difference-between-push-pull-marketing-31806.html [accessed 2017-05-01]

46. Rothman AJ, Salovey P. Shaping perceptions to motivate healthy behavior: the role of message framing. Psychol Bull 1997 Jan;121(1):3-19. [Medline: 9000890]

47. Gerend MA, Shepherd JE. Using message framing to promote acceptance of the human papillomavirus vaccine. Health Psychol 2007 Nov;26(6):745-752. [doi: 10.1037/0278-6133.26.6.745] [Medline: 18020847]

48. Abhyankar P, O'Connor DB, Lawton R. The role of message framing in promoting MMR vaccination: evidence of a loss-frame advantage. Psychol Health Med 2008 Jan;13(1):1-16. [doi: 10.1080/13548500701235732] [Medline: 18066916]

49. Gainforth HL, Cao W, Latimer-Cheung AE. Message framing and parents' intentions to have their children vaccinated against HPV. Public Health Nurs 2012 Nov;29(6):542-552. [doi: 10.1111/j.1525-1446.2012.01038.x] [Medline: 23078425]

50. Frew PM, Owens LE, Saint-Victor DS, Benedict S, Zhang S, Omer SB. Factors associated with maternal influenza immunization decision-making. Evidence of immunization history and message framing effects. Hum Vaccin Immunother 2014;10(9):2576-2583 [FREE Full text] [doi: 10.4161/hv.32248] [Medline: 25483468]

51. Hendrix KS, Finnell SM, Zimet GD, Sturm LA, Lane KA, Downs SM. Vaccine message framing and parents' intent to immunize their infants for MMR. Pediatrics 2014 Sep;134(3):e675-e683 [FREE Full text] [doi: 10.1542/peds.2013-4077] [Medline: 25136038]

52. Reno JE, O'Leary ST, Pyrzanowski J, Lockhart S, Thomas J, Dempsey AF. Evaluation of the implementation of a multicomponent intervention to improve health care provider communication about human papillomavirus vaccination. Acad Pediatr 2018;18(8):882-888. [doi: 10.1016/j.acap.2018.08.004] [Medline: $\underline{\text { 30107236] }}$

53. Gagneur A, Lemaître T, Gosselin V, Farrands A, Carrier N, Petit G, et al. A postpartum vaccination promotion intervention using motivational interviewing techniques improves short-term vaccine coverage: PromoVac study. BMC Public Health 2018 Jun 28;18(1):811 [FREE Full text] [doi: 10.1186/s12889-018-5724-y] [Medline: 29954370]

54. Kahan D. Fixing the communications failure. Nature 2010 Jan 20;463(7279):296-297. [doi: 10.1038/463296a]

55. Vinson DE, Scott JE, Lamont LM. The role of personal values in marketing and consumer behavior. J Marketing 2018 Nov 28;41(2):44-50. [doi: 10.1177/002224297704100215]

56. Edwards JR, Cable DM. The value of value congruence. J Appl Psychol 2009 May;94(3):654-677. [doi: 10.1037/a0014891] [Medline: 19450005$]$

57. McLeod MS. Values, communication behavior, and political participation. Political Commun 2001 Jul;18(3):273-300. [doi: 10.1080/10584600152400347]

58. Sweeney AM, Moyer A. Self-affirmation and responses to health messages: a meta-analysis on intentions and behavior. Health Psychol 2015 Feb;34(2):149-159. [doi: 10.1037/hea0000110] [Medline: 25089345]

59. Ryan RM, Deci EL. Self-determination theory and the facilitation of intrinsic motivation, social development, and well-being. Am Psychol 2000 Jan;55(1):68-78. [Medline: 11392867]

60. Falk EB, O'Donnell MB, Cascio CN, Tinney F, Kang Y, Lieberman MD, et al. Self-affirmation alters the brain's response to health messages and subsequent behavior change. Proc Natl Acad Sci U S A 2015 Feb 17;112(7):1977-1982 [FREE Full text] [doi: 10.1073/pnas.1500247112] [Medline: 25646442]

61. Kelly MP, Heath I, Howick J, Greenhalgh T. The importance of values in evidence-based medicine. BMC Med Ethics 2015 Oct 12;16(1):69 [FREE Full text] [doi: 10.1186/s12910-015-0063-3] [Medline: 26459219]

62. Attwell K, Freeman M. I Immunise: an evaluation of a values-based campaign to change attitudes and beliefs. Vaccine 2015 Nov 17;33(46):6235-6240 [FREE Full text] [doi: 10.1016/j.vaccine.2015.09.092] [Medline: 26458802]

63. Flannigan L, Orenstein WA, Bednarczyk R. Personal values as mediators of receipt of non-influenza childhood vaccines. Open Forum Infect Dis 2017;4(1):S514. [doi: 10.1093/ofid/ofx163.1335]

64. Lane S, MacDonald NE, Marti M, Dumolard L. Vaccine hesitancy around the globe: analysis of three years of WHO/UNICEF Joint Reporting Form data-2015-2017. Vaccine 2018 Jun 18;36(26):3861-3867 [FREE Full text] [doi: 10.1016/j.vaccine.2018.03.063] [Medline: 29605516]

65. Marti M, de Cola M, MacDonald NE, Dumolard L, Duclos P. Assessments of global drivers of vaccine hesitancy in 2014-Looking beyond safety concerns. PLoS One 2017;12(3):e0172310 [FREE Full text] [doi: 10.1371/journal.pone.0172310] [Medline: 28249006]

\section{Abbreviations}

KPCO: Kaiser Permanente Colorado

MI: motivational interviewing

PVVS: Parental Vaccine Value Scale 
SUS: System Usability Scale

TPB: Theory of Planned Behavior

VAB: Values-Attitudes-Behavior

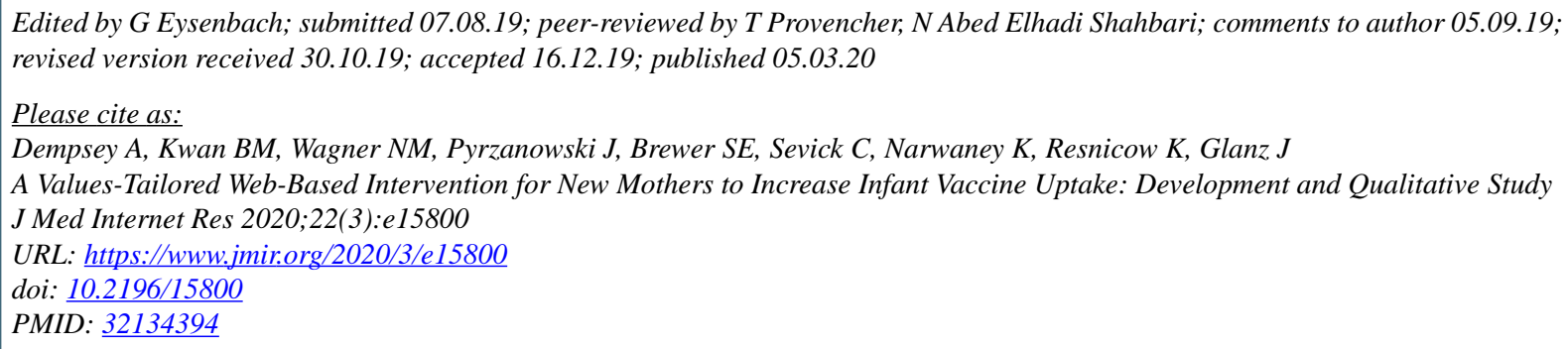

(C)Amanda Frisch Dempsey, Bethany M Kwan, Nicole M Wagner, Jennifer Pyrzanowski, Sarah E Brewer, Carter Sevick, Komal Narwaney, Kenneth Resnicow, Jason Glanz. Originally published in the Journal of Medical Internet Research (http://www.jmir.org), 05.03.2020. This is an open-access article distributed under the terms of the Creative Commons Attribution License (https://creativecommons.org/licenses/by/4.0/), which permits unrestricted use, distribution, and reproduction in any medium, provided the original work, first published in the Journal of Medical Internet Research, is properly cited. The complete bibliographic information, a link to the original publication on http://www.jmir.org/, as well as this copyright and license information must be included. 